\title{
SynRec: A Prediction Technique using Collaborative Filtering and Synergy Score
}

\author{
Nupur Kalra, Deepak Yadav, Gourav Bathla
}

\begin{abstract}
Recommender systems are techniques designed to produce personalized recommendations. Data sparsity, scalability cold start and quality of prediction are some of the problems faced by a recommender system. Traditional recommender systems consider that all the users are independent and identical, its an assumption which leads to a total ignorance of social interactions and trust among user. Trust relation among users ease the work of recommender systems to produce better quality of recommendations. In this paper, an effective technique is proposed using trust factor extracted with help of ratings given so that quality can be improved and better predictions can be done. A novel-technique has been proposed for recommender system using film-trust dataset and its effectiveness has been justified with the help of experiments.
\end{abstract}

Keywords: collaborative filtering, Recommender system, social trust, synergy score.

\section{INTRODUCTION}

A great change has been observed in the last decade in the field of e-commerce sites and development of social networks. Recommender systems is an intelligent approach to make a user experience better and helpful. Ecommerce sites like Flipkart, Amazon, Netflix are ruling the world with a huge variety of products and their services. Rating and reviews come along and play a major role in recommendations. For example Amazon uses collaborative filtering technique to provide recommendations, helps the user to filter the items as per his/her convince using its past history and ratings to the item. Quality of RS is determined by the recommendation it provides and how it deals with the user's behavior [1,2]. This paper focus on improving the quality addressing certain issues like cold start with the help of rating prediction. It's a complex process which combines consideration of many features of the items and information about the users.

Social media or social networking sites like Facebook, Twitter are great platforms where a user can connect with other users, can communicate on basis of trust and similar likes and dislikes. It results in producing large scale of data called big data. To analyze this big data which is increasing at every hour is a complex task, some tools like apache Hadoop, Apache spark and Mapreduce implementation are used to process the data.[3]Items are suggested by the recommendation system to the user by estimating the ratings user would give to the item.[4] Recommendations system

Revised Manuscript Received on July 22, 2019.

Nupur Kalra, CSE Department, Chandigarh University, Mohali, India. Deepak Yadav, CSE Department, Chandigarh University, Mohali, India

Gourav Bathla, CSE Department, Chandigarh University, Mohali, India. have many applications like travel and tourism, films, series, hotels, books etc. the process to estimate the ratings can be performed by using some techniques available in machine learning. There are three main basic approaches for recommender system.

1. Content based

2. Collaborative filtering

3. Hybrid based

Trust and similarity score is a boundless addition to the new approaches of recommender systems. It is believed that users prefers to receive a recommendation which is connected to the people they trust in one form or other. Trust based approaches are performing well as compared to using only similarity rating $[1,2,5]$. In this paper, Synergy score is introduced and a method is purposed to calculate it with the help of ratings given by the user. It helps to improve the quality of recommendations. SynRec attempts to work on problems like cold start, sparsity, scalability. They occur due to the lack of data or information about the user or item. Such conditions decreases the quality of recommendation which simultaneously effects the user experience. SynRec is a recommender system which predicts the ratings for a particular user and item to give recommendations. Relationship between the users is depicted with the help of synergy score, it's a score calculated on the basis of ratings given by the users to the items. It helps to normalize the problem of cold start and sparsity where we do not have proper rating or reviews to predict ratings and provide recommendations. The technique suggested has been tested on real life dataset Film-trust to show its improvements among other techniques.

\section{LITERATURE SURVEY}

According to [1] researchers these days are discussing about big data and social networks. There main aim is to extract patterns and relationships among data to provide better services. Author has discussed content based and collaborative filtering under the influence of social trusts using social network graphs.

A novel recommendation technique has been proposed by using item-rating matrix, calculating similarity among pairs using pearson correlation coefficient. Experimental analysis has been performed and effective improvements are observed as compared to trust walker list and top-n recommendation. 
Trust factor seen among users improves recommendations. Item based top-N recommendations has been used in[2] results given based on item correlation computation among all users. This paper includes four combined knowledge attack methods are proposed to determine that aggregated knowledge can be correctly inferred by attacking item based top-n recommender systems. Item based top-n recommendation algorithm implementation on fundan BBS, a famous social network among Chinese universities with $60 \mathrm{~K}$ users. The performance of the proposed technique is assessed in two characteristics aggregated to protect knowledge and recommendation accuracy loss. In [3] author has described the impact of social media on big data collectively calling it social big data. Several methodologies such as Mapreduce, tools to process big data such as apache Hadoop, apache spark etc. its application like marketing, crime analysis, epidemic intelligence are given. Issues emerging with the increasing data such as privacy, streaming of online and offline data. [5] states that collaborative filtering method is widely used and accepted by recommender systems which generate recommendation based on ratings given by like minded people but it comes with its own problems such as data sparsity, cold start.

They occur due to less ratings or no rating at all. Author has presented a model based on collaborative filtering method along with a novel graph clustering algorithm and trust stated. Experimental analysis has been performed on three real world datasets to show that the method proposed outperforms several other systems. Where as in [7] it states a review of recommendation systems, trust reputation systems and their combinations. Use of trust has emerged as a new way to find better recommendation. [9]- states data sparsity, scalability, prediction quality are some of the common problems faced by the recommender systems using collaborative filtering. It contains social recommendation framework which includes social network matrix factorization, user item matrix factorization. Experimental analysis has been performed on Epinions dataset comparing its algorithm with the other algorithms such as maximum margin matrix factorization, probabilistic matrix factorization and constrained probabilistic matrix factorization. They performed social analysis based on efficiency to decrease data sparsity and increase quality of predictions. Recommendation models regularized with user trust is in very much trend.[10]

The work conducted by the author shows that trust and rating compliments each other. Using four datasets they have conducted an empirical trust analysis, impact of different parameters and their comparison in trust degrees. this approach is suitable for large datasets like Epinions, Flixter etc using the scalability of trust SVD in terms of training time. In [11] novel approach by integrating sparse rating data given by users and social trust network effected by sparsity among other users. Datasets like Epinions, Douban and Flixter to validate on different parameters like cold start, rank based metrices. It's a more general and conforming framework referred as trustPMF to give a probabilistic view of trustee, truster, trustPMF. to enhances the quality of recommendation. [12] states a kind of model-based recommendation algorithms that first finds the similarities between the various items and then uses them to identify the set of items to be recommended to the user. The main steps in this class of algorithms are the method used to calculate the similarity among the items, and the method used to combine these similarities to find the similarity between a basket of items and a candidate recommender item experimental analysis on eight real datasets shows that these item-based algorithms are up to two times faster than the traditional user-neighborhood based recommender systems and provide recommendations with better quality.[20] states a traditional trust node method for calculating trust and some extra information to train the data. Node logistic regression model is used and collaborative filtering algorithm to find node trust value. Simulation is performed to verify the improved method. Results show better prediction accuracy of node trust value. [28] states a new method for top-k recommendation system called BTrank.

It integrates the trust model and rating to sort the system which significantly improves the quality of recommendations. [29] states that the rate of coverage shows a monotonous decrease in trend as the number of clusters are increasing idea of a 2D-Graph clustering method for subdividing a novel 2D-Graph in union with the minimum acceptable coverage rate standard. To reduce the negative impact of clustering with trust, Pearson is used for similarity in order to construct weighted 2D-Graph. The experimental on two real datasets shows that the proposed method performs better than other methods for Cold Start users with better accuracy and coverage.

Table 1 Represents a feature-wise comparison of different existing approaches studied during the research and along with the proposed technique SynRec. Existing approaches like matrix factorization, Social network based item recommendation etc. have tried to work on issues like cold start, scalability yet they have not been completely resolved and are not able to perform satisfactory. SynRec has dealt with every measure and have performed well with an improvement in accuracy.

\section{RECOMMENDATION TECHNIQUES}

Traditional recommender systems are basically content based and collaborative filtering method in which user's past history of ratings, behavior are considered. Recommendations can be personalized and non-personalized in approach as per user's aspect. Personalized recommendations are given a list of top recommended items on the site. The product can be suggested based on user's past behavior analysis or a demographic suggestion given by other user. Where as non-personalized are easy to generate, they are independent of user's behavior. They are automatically generated and needs user's effort to find best for him $[8,16,24]$.

A. Collaborative filtering- It uses the ratings or numerical data given by the user, available in the form of historical data in user profile. It is considered one of the most basic and widely used technique to give suggestions and make predictions regarding a product as per user preference. Collaborative filtering is usually applied on large datasets. 
Table 1: Comparison of different approaches

\begin{tabular}{|c|c|c|c|c|c|c|}
\hline Author & $\begin{array}{l}C \\
o l \\
d \\
s t \\
a r \\
t\end{array}$ & $\begin{array}{l}\text { Spa } \\
\text { rsit } \\
y\end{array}$ & $\begin{array}{l}\text { Scal } \\
\text { abilit } \\
y\end{array}$ & $\begin{array}{l}\text { Soc } \\
\text { ial } \\
\text { trus } \\
t\end{array}$ & $\begin{array}{l}\text { Pr } \\
\text { ed } \\
\text { ict } \\
\text { io } \\
n\end{array}$ & Technique \\
\hline [2] & $\checkmark$ & $x$ & $x$ & $\checkmark$ & $\checkmark$ & $\begin{array}{l}\text { Top-N } \\
\text { recommen } \\
\text { dation }\end{array}$ \\
\hline [4] & $x$ & $\checkmark$ & $x$ & $\checkmark$ & $\checkmark$ & $\begin{array}{l}\text { A latent } \\
\text { social trust } \\
\text { network } \\
\text { model }\end{array}$ \\
\hline [5] & $\checkmark$ & $\checkmark$ & $\checkmark$ & $x$ & $\checkmark$ & $\begin{array}{l}\text { Clustering } \\
\text { Algorithm } \\
\text { Trust } \\
\text { statement }\end{array}$ \\
\hline [7] & $\checkmark$ & $\checkmark$ & $x$ & $\checkmark$ & $\checkmark$ & $\begin{array}{l}\text { Social } \\
\text { Network-b } \\
\text { ased item } \\
\text { recommen } \\
\text { dation }\end{array}$ \\
\hline [9] & $\checkmark$ & $\checkmark$ & $\checkmark$ & $x$ & $\checkmark$ & $\begin{array}{l}\text { Analysis } \\
\text { approach } \\
\text { for social } \\
\text { recommen } \\
\text { dation }\end{array}$ \\
\hline [10] & $\checkmark$ & $\checkmark$ & $x$ & $x$ & $\checkmark$ & $\begin{array}{l}\text { Extended } \\
\text { form of } \\
\text { SVD++ }\end{array}$ \\
\hline [11] & $\checkmark$ & $\checkmark$ & $x$ & $\checkmark$ & $\checkmark$ & $\begin{array}{l}\text { Matrix } \\
\text { Factorizati } \\
\text { on }\end{array}$ \\
\hline $\begin{array}{l}\text { Propo } \\
\text { sed } \\
\text { Techni } \\
\text { que }\end{array}$ & $\checkmark$ & $\checkmark$ & $\checkmark$ & $\checkmark$ & $\checkmark$ & SynRec \\
\hline
\end{tabular}

B. Content based filtering- It focuses on the features of item. It creates a user profile based on the previous reviews of the users and also in accordance with similar kind of items. It extracts information from the item and from the dynamic user. It provides recommendation according to user's field of interests and open to adaptation of user's like or dislikes. It helps to remove sparsity which is common in collaborative filtering.

C. Hybrid Based-Its main aim is to overcome the problems faced in collaborative and content based filtering . it contains techniques like weighted switching, mixed, feature combinations.

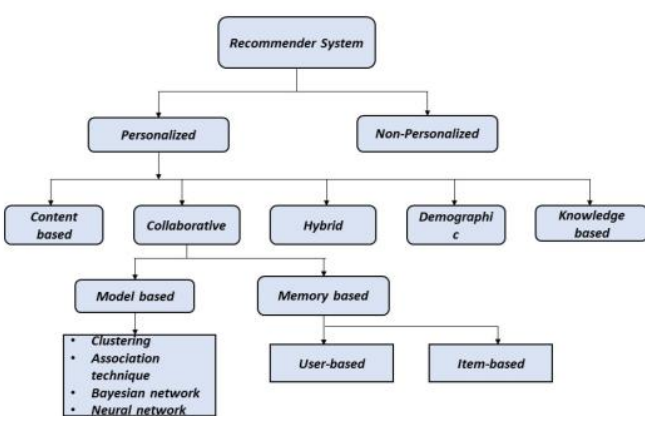

Fig 1: Classification of recommender system

\section{Challenges in Recommender systems}

- Cold start problem -at early stages of an item, when it is added to the dataset, there is little information or no information available to use by system.

- Accuracy suggestions- suggestions given should be appropriate, it should be useful to the user. Lower accuracy in prediction may lead to loose the interest of user.

- Changing dataset- addition of new items on daily purpose will increase the size and will test the algorithm to predict better. This makes the efficiency go down of the system.[27]

- Sparsity -usually user purchase items online but do not rate them or give reviews, which makes it to difficult find similar users or locate neighbors

- Privacy - data collected for analysis or being used for commercial purpose should be kept safe .value the risk of personal data of the users, modification of the data should be avoided $[8,11,17]$.

- Scalability and big data- huge amount of data is difficult to process. it may compromise between the performance and prediction quality of a RS.[29].

E. Trust relationship- It is one of the most influential information and trusted social information to use in recommender system. for example we ask our friends for a product recommendation and we trust their advice more than any other individual we do not connect to. Very few trust based models are available in this field.

These explanations lead us to consider both explicit and implicit influence of the item rating and user relations. Explicit trust are real values of ratings and implicit is who reviewed what item, what ratings, who trust whom. Implicit trust is more helpful in recommendations and predictions. Trust is very sparse but it plays a vital role and its complimentary. Reliability and decision trust are two type of trust has been observed. Reliability trust is one in which a user expects from another user to perform a certain function which is for welfare where as in Decision trust is dependent on situations with a feeling of security, consequences may lead to negative outcomes $[1,5,6,10,18]$. 
General Algorithms-used in recommender system are example Matrix factorization [9,17], SVD- singular value decomposition [10], KNN model [28], Clustering [5,6], top-N recommendation $[2,19]$

\section{PROPOSED METHOD}

A. Proposed method-Recommendation are provided on the basis of rating given to the item by a user. But the results obtained using user-item ratings are not satisfying. If we consider Trust factor with user -rating it adds more value to the final results. In this we paper we are presenting a new method which is an amalgamation of a collaborative filtering based recommender system and social trust. This approach not only giving better results in predicting the ratings as compared to other state of art but also introducing a new term Synergy score $\left(\mathrm{Sy}_{\mathrm{i}}\right)$ which is a non-binary form of trust score. Earlier we were only aware of binary trust score which is either 0 or 1 .

Case1: when user $\mathrm{A}$ is consuming item 5, what rating might $\mathrm{A}$ give to item 5?A naive approach is to take a global Average for that product which will fill it with $(5+3.5) / 2=4.25$. There is no relation or behaviour defined for the user ' $A$ ' on basis of which we can predict it. User ' $A$ ' has nothing to do with what user may rate. We are not sure if the user will behave in that way.

\begin{tabular}{|l|l|l|}
\hline USER & ITEM & RATING \\
\hline A & 5 & $?$ \\
\hline B & 5 & 5.0 \\
\hline C & 5 & 3.5 \\
\hline
\end{tabular}

Fig 2: case 1

Case2: A and B are friends, Let's assume they have similar behaviour and have trust on each other. As we see, A has a trust on $\mathrm{B}$ so the behaviour of $\mathrm{A}$ is like $\mathrm{B}$ not $\mathrm{C}$ so the rating filled will be 5.0 But if there are too many friends of A having trust but have different opinion on one item? A naive solution is to consider only trusted users and take an average of them. That will fill it with $(5.0+3.0+2.0+1.0) / 4=2.4$

\begin{tabular}{|l|l|l|l|}
\hline USER & ITEM & RATING & TRUST \\
\hline A & 7 & & NULL \\
\hline B & 7 & 5.0 & 1 \\
\hline C & 7 & 3.0 & 0 \\
\hline
\end{tabular}

Fig 3: case2(a)

\begin{tabular}{|l|l|l|l|}
\hline USER & ITEM & RATING & TRUST \\
\hline A & 7 & & NULL \\
\hline B & 7 & 5.0 & 1 \\
\hline C & 7 & 3.0 & 0 \\
\hline D & 7 & 2.0 & 1 \\
\hline E & 7 & 1.0 & 1 \\
\hline
\end{tabular}

Fig 4: case 2(b)

Case 3: What if $\mathrm{A}$ and $\mathrm{B}$ are close friends and $\mathrm{A}$ also has other friends? A solution is to have A's behaviour more like B because of having a relationship perk and still average suggestions of others. But if there are many other relations of A along with these, also have different opinion on item?

\begin{tabular}{|l|l|l|l|l|}
\hline $\begin{array}{l}\text { USE } \\
\mathbf{R}\end{array}$ & $\begin{array}{l}\text { ITE } \\
\mathbf{M}\end{array}$ & $\begin{array}{l}\text { RATIN } \\
\mathbf{G}\end{array}$ & $\begin{array}{l}\text { CloseFriend } \\
\mathbf{s}\end{array}$ & $\begin{array}{l}\text { TRUS } \\
\mathbf{T}\end{array}$ \\
\hline $\mathrm{A}$ & 7 & & NULL \\
\hline $\mathrm{B}$ & 7 & 5.0 & yes & 1 \\
\hline C & 7 & 3.0 & no & 0 \\
\hline $\mathrm{D}$ & 7 & 2.0 & no & 1 \\
\hline $\mathrm{E}$ & 7 & 1.0 & no & 1 \\
\hline
\end{tabular}

Fig 5: case 3

B. Synergy score- Synergy score is the concept of weighted score calculated with the help of rating given by the similar user and the subject user. This score will define the amount of trust a subject user has with other users. Higher is the synergy score more is the trust. Synergy will present how the much the other user behaves like our subject user.

To calculate synergy score between two users:

$$
S y_{o s}=\sum_{i}^{n}(2.5-a b s(R o-R s)
$$

Where $\boldsymbol{R}_{\boldsymbol{o}}$ is the rating given by other user, $\boldsymbol{R}_{\boldsymbol{s}}$ is rating given by subject user and $\boldsymbol{S y _ { o s }}$ is the synergy score of other users w.r.t subject user. Synergy will depict how much the subject user behaves similar to other users.

We can visualize the relation of subject user with other users as given in the following image. $S$ in centre being Subject user and the closer other users are more is the synergy score between them. For example user with synergy score 27 has more similar behaviour with subject(S) as compared to user with synergy score 5 . 
C. Recommendation-Now that we have synergy score of our subject user with every other user, we rank the movies that are unwatched by the subject and can be recommended. This scoring process is called normalised rating.

$$
\text { Normalised Rating }=\sum_{1}^{n} \quad S y i * R k
$$

Where $\boldsymbol{S} \boldsymbol{y}_{\boldsymbol{i}}$ is synergy score with other user $i, \boldsymbol{R}_{\boldsymbol{i}}$ is rating given for movie $k$ and $\mathrm{n}$ is the $\mathrm{x} \%$ of total other users having positive synergy score. we order our movies based on the normalised rating to recommend to our subject user.

D. Prediction- Using synergy score we can define the behaviour of the users but we have used this score further to predict the rating which might be given by the subject user. It's a prediction process, using this technique we have tried to improve the accuracy rate among the actual rating and predicted ratings.

Predicted - Rating $\left(R^{\prime} i\right)=\sum_{1}^{n} S y_{i} * R_{k} / \sum_{i}^{n} S y_{i}$

We have considered $100 \%$ users having synergy score $>0$ for prediction. We can also use top $90 \%$ or $50 \%$ for empirically proven improved prediction using the same formulation. where $\boldsymbol{R}_{\boldsymbol{i}}$ is the predicted rating.

\section{E. Algorithm}

Input: user_id (subject user) for recommendations and prediction.

Output: List of recommended movies based on synergy score, prediction of rating and calculating error rate.

1. Input the user-id of the subject user.

2. Find similar users w.r.t subject user.

3. Calculate the synergy score of subject user with other users.

$$
S y_{o s}=\sum_{i}^{n}(2.5-a b s(R o-R s))
$$

4. Predict rating using sum of the product of synergy score and rating divided by sum of synergy score.

$$
R_{i=}^{,} \sum_{i}^{n} S_{y i} * R k / \sum_{i}^{n} S_{y i}
$$

5. Calculate the MAE to analyse the error rate among predicted rating and actual ratings.

$$
M A E=\sum_{i}^{N} R^{\prime} i-R i / N
$$

\section{EXPERIMENT ANALYSIS}

In this section, we conduct an experiment analysis on the proposed technique to investigate its effectiveness as compared to other approaches.
A. Dataset- Dataset used is Film-trust. The main source of this data set is librec documentation. It acquires three main attributes user-id, movie-id and ratings given by the user. Total data is 35,497, with approximately 1500 users.

\begin{tabular}{|l|l|}
\multicolumn{2}{|c}{ Table 2: Description of dataset } \\
\hline Features & FilmTrust \\
\hline \#users & 1,508 \\
\hline \#items & 2,071 \\
\hline \#ratings & 35,497 \\
\hline
\end{tabular}

B. Tools-IntelliJ, Programming is done in java using IntelliJ. IntelliJ IDEA, a Java integrated development environment to develop software. It is developed by JetBrains. It is available as an Apache 2 Licensed community edition, and in a commercial edition. Both can be used for commercial development.

C. Evaluation metrics- We have adopted MAE mean absolute error where $\mathrm{N}$, the number of test cases. Lesser the values of MAE indicates the more predictive accuracy of the system or technique. A good recommender should give less error for better efficiency.

$$
M A E=\sum_{i}^{\mathbb{N}} R_{i}^{\prime}-R i / N
$$

D. Experimental setup and Results-In this section we conduct experiment to check the quality of recommendation and predictions given by our method. To comparatively evaluate the proposed technique SynRec, we have selected two other methods for recommendation Trusted social network based Recommendation system [14], in which users with similar preferences are searched from user social network to give recommendation based on trusted networks. Every feedback given by the user after a transaction enhances the trust relation among users. They use Pearson's correlation coefficient for similar neighbours and Clique Perlocation Method (CPM) to find community structures. And a collaborative filtering algorithm with combination of explicit and implicit trust, in which author has used both explicit and implicit trust to produce better recommendation and predict trust score among users called EImerge. It works to alleviate problems of cold-start and sparsity observed in recommender systems. They have used Pearson's correlation coefficient to find implicit trust among neighbours and trust propagation of rating prediction [15]. We have used Film-trust dataset, is available at librec as its main source. It was established in June 2011. Its main aim is to contribute to research work. It contains 1508 users, 2071 items and 35,497 ratings. Simulated experiments have been performed to evaluate the SynRec method for quality of recommendation and accuracy of predicted rating using IntelliJ on configured platform

Windows 10, intel i7 700HQ,2.8GHz CPU,8GB RAM. 


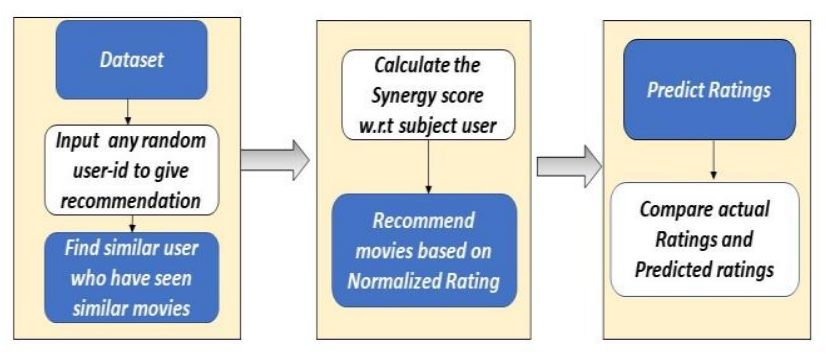

Fig 7: Flow diagram of the proposed approach

The Performance of the SynRec, Trust community based algorithm and EImerge on the basis of MAE. We have selected $80 \%$ data for training and rest $20 \%$ of it for the testing purpose.

In Table 3 shows the accuracy (MAE) results for three different techniques Trust Community Based Algorithm, EImerge and proposed technique SynRec. SynRec has improved its accuracy in prediction by $2.2 \%$ w.r.t to EImerge algorithm and $11.6 \%$ improvement has been observed by Trust community based algorithm. It is also validated with the help of MAE. It can be concluded that smaller changes in trust method can improve accuracy of recommendation and prediction and provide better results.

Table 3: Accuracy of prediction For different algorithms.

\begin{tabular}{|l|c|}
\hline Approaches & MAE \\
\hline Trust community based Algorithm & .7931 \\
\hline EImerge & .7173 \\
\hline SynRec & .7009 \\
\hline
\end{tabular}

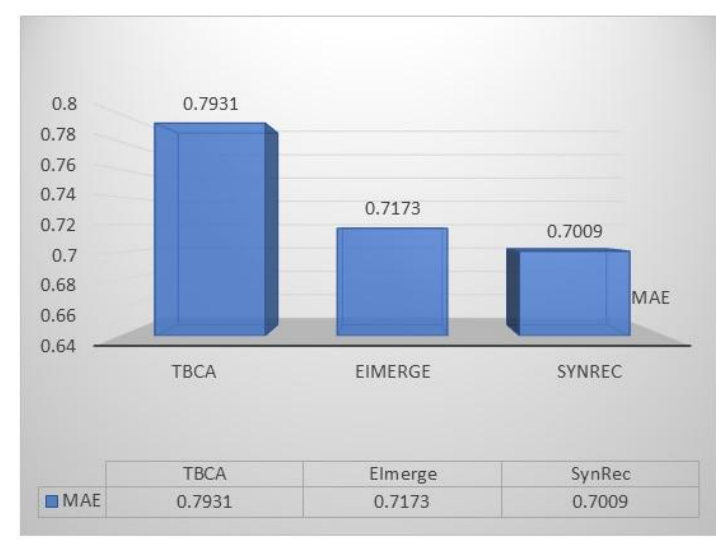

Fig 8: Graphical presentation of MAE of different algorithm.

In Table 4, we represent the variation in MAE as by varying the percentage of top similar users in the test case, which is referred as threshold value of similar users

Table 4: variation of MAE with the changing threshold value.

\begin{tabular}{|l|l|l|l|l|l|}
\hline $\begin{array}{l}\text { Thresh } \\
\text { hold of } \\
\text { similar } \\
\text { users }\end{array}$ & $\begin{array}{l}100 \\
\%\end{array}$ & $90 \%$ & $70 \%$ & $50 \%$ & $30 \%$ \\
\hline MAE & .700 & $\begin{array}{l}.7009 \\
325\end{array}$ & $\begin{array}{l}.700 \\
8777\end{array}$ & $\begin{array}{l}.6997 \\
221\end{array}$ & .696 \\
& 9354 & 3150 \\
\hline
\end{tabular}

\section{CONCLUSION AND FUTURE DIRECTION}

Technique proposed SynRec is a recommendation technique using collaborative filtering and. It has significantly shown improvements in prediction accuracy of the ratings as compared to some other techniques purposed in the past. Traditional recommender systems shows that users are independent of each other and report certain issues. In this paper we have formulized Synergy score among users which has a great influence on the quality of Recommendation, cold start, sparsity. Experiment analysis on FilmTrust proves that recommendation is improved by using our proposed approach. MAE is improved which is demonstrated in Experiment analysis. Further improvements are possible in this technique by improving other measures. Evaluation metrics such as precision, recall and RMSE can be used. Datasets such as Epinions, MovieLens can be used for experiment.

\section{REFERENCES}

1. G. Bathla," A graph-based model to improve social trust and influence for social recommendation," The Journal of Supercomputing, 2017,1-19.

2. D.Li., “ Item-based top-N recommendation resilient to aggregated information revelation," Knowledge-Based Systems, 67,2014, 290-304.

3. G. Bello-Orgaz "Social big data: Recent achievements and new challenges". Information Fusion, 28, 2016,45-59

4. X.Yang, "A product recommendation approach based on the latent social trust network model for collaborative filtering," In 2016 IEEE International Conference on Software Quality, Reliability and Security Companion $(Q R S-C)$,2016,pp. 178-185. IEEE.

5. P.Moradi, " A reliability-based recommendation method to improve trust-aware recommender systems," Expert Systems with Applications, 42(21), 2015,7386-7398.

6. P.Moradi," An effective trust-based recommendation method using a novel graph clustering algorithm," Physica A: Statistical Mechanics and its Applications, 436, 2015,462-481.

7. M.G.Ozsoy, "Trust based recommendation systems," In 2013 IEEE/ACM International Conference on Advances in Social Networks Analysis and Mining (ASONAM 2013),pp. 1267-1274, IEEE.

8. N.Sodera, " Open problems in recommender systems diversity," In 2017 International Conference on Computing, Communication and Automation (ICCCA), 2017 pp. 82-87, IEEE.

9. H.Ma, "Sorec: social recommendation using probabilistic matrix factorization," In Proceedings of the 17th ACM conference on Information and knowledge management ,2008,pp. 931-940, ACM.

10. G.Guo, " A novel recommendation model regularized with user trust and item ratings" IEEE Transactions on Knowledge and Data Engineering, 28(7), 2016,1607-1620.

11. B.Yang,"Social collaborative filtering by trust," IEEE transactions on pattern analysis and machine intelligence, 39(8), 2017,1633-1647.

12. M,Deshpande," Item-based top-n recommendation algorithms," ACM Transactions on Information Systems (TOIS), 22(1),2004, 143-177.

13. P.Sun, (2018, May)" Research of Personalized Recommendation Algorithm Based on Trust and User's Interest," In 2018 International Conference on Robots \& Intelligent System (ICRIS) 2018,pp. 153-156, IEEE.

14. W.Wenjuan, (2015, July)"A personalized recommendation strategy based on trusted social community," In 2015 10th International Conference on Computer Science \& Education (ICCSE), 2015,pp. 496-499, IEEE.

15. H.Zhao, "A New Collaborative Filtering Algorithm with Combination of Explicit Trust and Implicit Trust," In 2018 13th International Conference on Computer Science \& Education (ICCSE),2018, pp. 1-5,IEEE.

16. F,Isinkaye," Recommendation systems: Principles, methods and evaluation," Egyptian Informatics Journal, 16(3), 2015,261-273.

17.M. Vlachos," Addressing Interpretability and Cold-Start in Matrix Factorization for Recommender Systems," IEEE Transactions on Knowledge and Data Engineering,2018. 
18. A.Davoudi, (2016, January)" Product rating prediction using trust relationships in social networks," In 2016 13th IEEE Annual Consumer Communications \& Networking Conference (CCNC),2016, pp. 115-118, IEEE

19. C.Park," Improving top-K recommendation with truster and trustee relationship in user trust network". Information Sciences, 374,2017, 100-114.

20. Y.Wang," A trust-based probabilistic recommendation model for social networks," Journal of Network and Computer Applications, 55, 2015,59-67.

21. J.Golbeck, "Filmtrust: Movie recommendations using trust in web-based social networks," In Proceedings of the IEEE Consumer communications and networking conference ,Vol. 96, No. 1,2016, pp. 282-286.

22. F.Walter," A model of a trust-based recommendation system on a social network," Autonomous Agents and Multi-Agent Systems, 16(1),2008, 57-74.

23. J.Herlocker ,"Evaluating collaborative filtering recommender systems," ACM Transactions on Information Systems (TOIS), 22(1),2004, 5-53.

24. R.Katarya, "An effective collaborative movie recommender system with cuckoo search," Egyptian Informatics Journal, 18(2),2017, 105-112.

25. N.de Mello, "Insights on social recommender system," In Proceedings of the Workshop on Recommendation Utility Evaluation: Beyond RMSE, at ACM RecSyS12,2012, pp. 33-38.

26. X.Wu," Data mining with big data," IEEE transactions on knowledge and data engineering, 26(1),2014 97-107.

27.R. Mukkamala," Fuzzy-set based sentiment analysis of big social data," In 2014 IEEE 18th International Enterprise Distributed Object Computing Conference ,2014, pp. 71-80, IEEE.

28. L.Xiong," An Approach for Top-k Recommendation Based on Trust Information," In 2017 IEEE 10th Conference on Service-Oriented Computing and Applications (SOCA), 2017,pp. 198-205, IEEE.

29.L. Sheugh," A novel 2D-Graph clustering method based on trust and similarity measures to enhance accuracy and coverage in recommender systems," Information Sciences, 432,2018, 210-230.

\section{Authors ProfiLe}

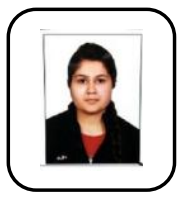

Nupur Kalra have completed BTech. From BKBIET, pilani. Pursuing Master of engineering in CSE from Chandigarh University, Mohali. Area of interest is machine learning and data analytics. Published a paper on movie recommender system using collaborative filtering. She can be contacted at nupurkalra2109@gmail.com.

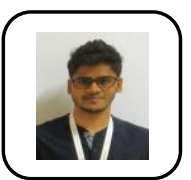

Deepak Yadav pursuing BE $\left(3^{\text {rd }} \mathrm{yr}\right)$ from Chandigarh University. Lead of Developer Student Club powered by Google Developers' Programme. His area of interest is Software engineering and Data science. A passionate and flexible Engineer having extensive experience in Software Engineering. He has trained and mentored more than 300 students in Java, Android and SE. He can be contacted at deepakyadav.j23@gmail.com.

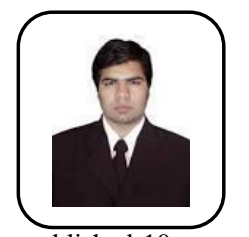

Gourav Bathla assistant professor at Chandigarh University. is working as Assistant Professor at Chandigarh University, Punjab, India. He has 12 years of teaching experince. He has completed M.E from Delhi College of Engieering. He is GATE qualified with All India Rank 59. He is an active researcher and published 10 research papers in reputed journals and 5 research papers in International conferences. His areas of interest are Big Data, Machine Learning, Deep Learning, Data analytics and Programming Langauges. He may be contacted at gouravbathla@gmail.com. 